\title{
Enhancement of symmetry-induced photoluminescence in bismuth tungstate microcrystals
}

\author{
Poliana Lima Rocha ${ }^{a}$, Diego Augusto Batista Barbosa ${ }^{\mathrm{b}}$, José Renato de Oliveira Lima ${ }^{\mathrm{a}}$, \\ Gilza Maria Piedade Prazeres ${ }^{c}$, Carlos William de Araujo Paschoal ${ }^{\mathrm{d}}$, Maximo Siu Li ${ }^{\mathrm{e}}$, \\ Elson Longo ${ }^{\mathrm{f}}$, Adeilton Pereira Maciel ${ }^{\mathrm{c}}$, Marcio Aurélio Pinheiro Almeida ${ }^{\mathrm{a}, *}$ \\ ${ }^{a}$ Coordenação de Ciência de Tecnologia, Universidade Federal do Maranhão, Campus do Bacanga, 65085-580 São Luis, MA, Brazil \\ b Departamento de Física, Universidade Federal do Maranhão, Campus do Bacanga, 65085-580 São Luis, MA, Brazil \\ ${ }^{c}$ Departamento de Química, Universidade Federal do Maranhão, Campus do Bacanga, 65085-580 São Luis, MA, Brazil \\ d Departamento de Física, Universidade Federal do Ceará, Campus do Pici, 65455-900 Fortaleza, CE, Brazil \\ e Instituto de Física de São Carlos-Universidade de São Paulo, CEP 13560-970 São Carlos, SP, Brazil \\ ${ }^{\mathrm{f}}$ Instituto de Química, UNESP-Universidade Estadual Paulista, 14800-900 Araraquara, SP, Brazil
}

\section{A R T I C L E I N F O}

\section{Article history:}

Received 2 June 2016

Received in revised form

27 July 2016

Accepted 10 August 2016

Available online 10 August 2016

Keywords:

Bismuth tungstate

Structural change

Photoluminescence

\begin{abstract}
A B S T R A C T
$\mathrm{Bi}_{2} \mathrm{WO}_{6}$ (BWO) powders synthesized via hydrothermal processes were studied in order to determine the effect of structure on photoluminescence (PL). BWO samples were characterized using X-Ray Powder Diffraction, UV-vis diffuse reflectance, field emission scanning electron microscopy, and PL intensity measurements. Orthorhombic structures were observed in all samples. A change in crystal microstructure and optical band gap was observed. The PL intensity obtained with different samples varied, with the highest intensity obtained with BWO fabricated with polyvinylpyrrolidone (PVP). Using Raman spectroscopy, a change in the microcrystal symmetry from $P 2{ }_{1} a b$ to $B 2 c b$ was observed and found to be responsible for the increase in photoluminescence.
\end{abstract}

(c) 2016 Elsevier B.V. All rights reserved.

\section{Introduction}

Metal tungstates have been the subject of much attention because of their large range of chemical and physical properties [1,2]. $\mathrm{Bi}_{2} \mathrm{WO}_{6}(\mathrm{BWO})$, in particular, has been widely investigated because of its interesting ferroelectric [3] and piezoelectric [4] properties, as well as its catalytic potential [5,6], optical properties [7], and magnetic characteristics [8]. It is crucial to investigate the photoluminescence (PL) properties of bismuth tungstates because these materials are promising candidates for application as optical scintillators $[7,9]$. Structural network reconfiguration is a potentially important way to increase the PL properties of inorganic solids. Reconfiguration can be achieved by altering the conditions during metal tungstate synthesis such as doping-level [10,11], synthesis methodology [12,13], and other factors [14]. Although it is known that the PL of metal tungstates is directly linked to $\mathrm{WO}_{4}{ }^{2-}$ electronic transitions, the creating of structural defects has also been proven to be a very important factor in the enhancement of PL properties of these materials [15]. These defects can be created using synthesis templates that have the ability to control the

\footnotetext{
* Corresponding author.

E-mail address: almeida.pinheiroa@gmail.com (M.A.P. Almeida).
}

formation of a particular structure [16,17]. Although previous investigations describe the PL properties of $\mathrm{Bi}_{2} \mathrm{WO}_{6}[7,18]$, the effect of template-modified structure on these properties has not previously been reported. Therefore, in this letter, the influence of sodium dodecyl sulfate (SDS) and polyvinylpyrrolidone (PVP) surfactant-assisted synthesis on PL properties of BWO microcrystals is investigated.

\section{Experimental}

\subsection{Synthesis of $\mathrm{Bi}_{2} \mathrm{WO}_{6}$ microcrystals}

$\mathrm{Bi}_{2} \mathrm{WO}_{6}$ samples were obtained by a previously reported surfactant-assisted hydrothermal method [6]. Typically, $1.0 \mathrm{mmol}$ $(0.3332 \mathrm{~g})$ of sodium tungstate dihydrate $\left(\mathrm{Na}_{2} \mathrm{WO}_{4} \cdot 2 \mathrm{H}_{2} \mathrm{O}, 99 \%\right.$ purity, Aldrich) and $2.0 \mathrm{mmol}(0.9898 \mathrm{~g})$ of bismuth nitrate (Bi $\left(\mathrm{NO}_{3}\right)_{3}, 99 \%$ purity, Aldrich) were added to $100 \mathrm{~mL}$ of surfactant solution (SDS, PVP). The mixture was stirred for $10 \mathrm{~min}$ and then transferred into a $150 \mathrm{~mL}$ Teflon-lined stainless autoclave, and kept at $180^{\circ} \mathrm{C}$ for $12 \mathrm{~h}$. After centrifuging, washing with deionized water, and drying in an oven at $60{ }^{\circ} \mathrm{C}$, pale yellow powder samples were obtained. The samples were called: sample S1 (without surfactant), sample S2 (SDS), sample S3 (PVP) and sample S4 (S3 
after annealing at $600{ }^{\circ} \mathrm{C}$ by $4 \mathrm{~h}$ ).

\subsection{Characterization}

X-ray powder diffraction (XRPD) patterns were obtained using a D/Max-2500PC diffractometer (Rigaku, Japan) with $\mathrm{Cu}-\mathrm{K} \alpha$ radiation ( $1.5406 \AA$ ) at $40 \mathrm{kV}$ and $150 \mathrm{~mA}$, between a $2 \theta$ angle range of $5-85^{\circ}$; these patterns were used to confirm the structures against those previously obtained [6]. Fourier transform Raman (FT-Raman) spectra were recorded using a Bruker-RFS 100 (Germany) spectrometer. Raman spectra were obtained using an $\mathrm{Nd}$ : YAG laser (1064 $\mathrm{nm})$, keeping the maximum output power at $100 \mathrm{~mW}$. The spectra were acquired in the range of $50-1000 \mathrm{~cm}^{-1}$. PL measurements were carried out using a Monospec 27 monochromator (Thermal Jarrel Ash, USA) coupled to a R446 photomultiplier. A krypton ion laser $(\lambda=350 \mathrm{~nm})$ was used as the excitation source; the maximum output power was maintained at $500 \mathrm{~mW}$. PL measurements were taken at room temperature.

\section{Results and discussions}

\subsection{Structural analysis}

The as-prepared BWO sample S1 obtained without surfactant exhibited the usual $P 2{ }_{1} a b$ orthorhombic phase according to the JCPDS card No 39-0256 [15], as did Sample S2, formed in the presence of SDS. Sample S3, formed in the presence of PVP, exhibited a slightly different structure (see Fig. 1(c)), as confirmed by Raman spectroscopy (see Fig. 2(d)). As discussed previously [6], the presence of PVP during synthesis induces a B2cb metastable structure, which can be transformed into the usual $P 2{ }_{1} a b$ structure by annealing (Fig. 2c). The Raman spectra of sample S4 (S3 after annealing), was shown to have a similar profile to samples S1 and $\mathrm{S} 2$, thus confirming the metastable nature of the $B 2 c b$ phase.

\subsection{Photoluminescence}

In previous research, the PL phenomenon of metal tungstates was ascribed only to charge-transfer transitions within the $\left[\mathrm{WO}_{4}\right]^{2-}$ complex in ordered systems [15]. However, some of these electronic transitions are forbidden by the selection rule [19]. Factors such as complex cluster vacancies, modified lattices, and/or

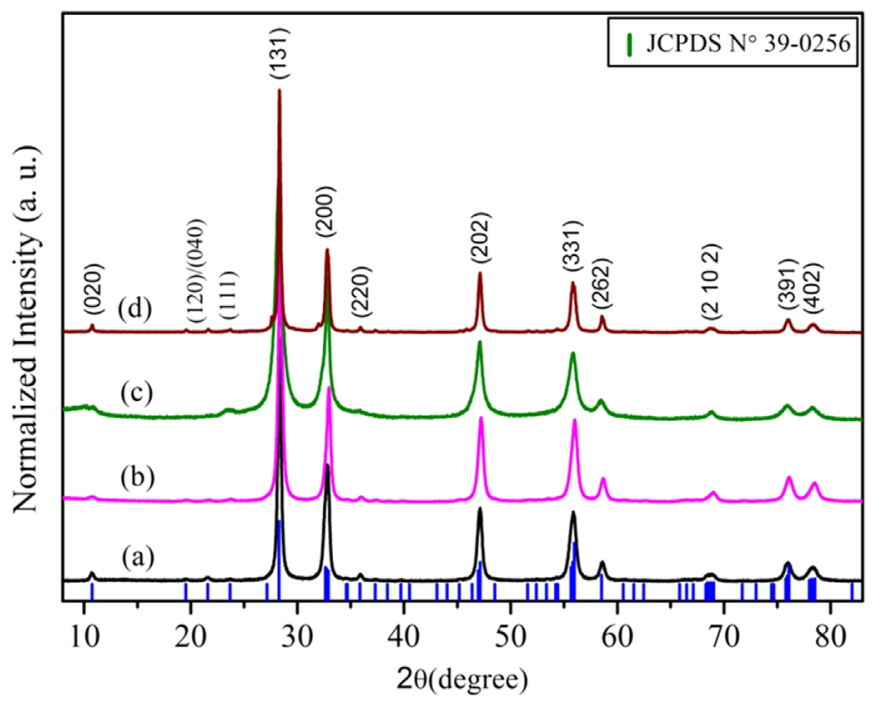

Fig. 1. XRPD patterns of the as-prepared BWO samples: (a) S1 (no surfactant), (b) S2 (SDS), (c) S3 (PVP), and (d) S4 (PVP after annealing).

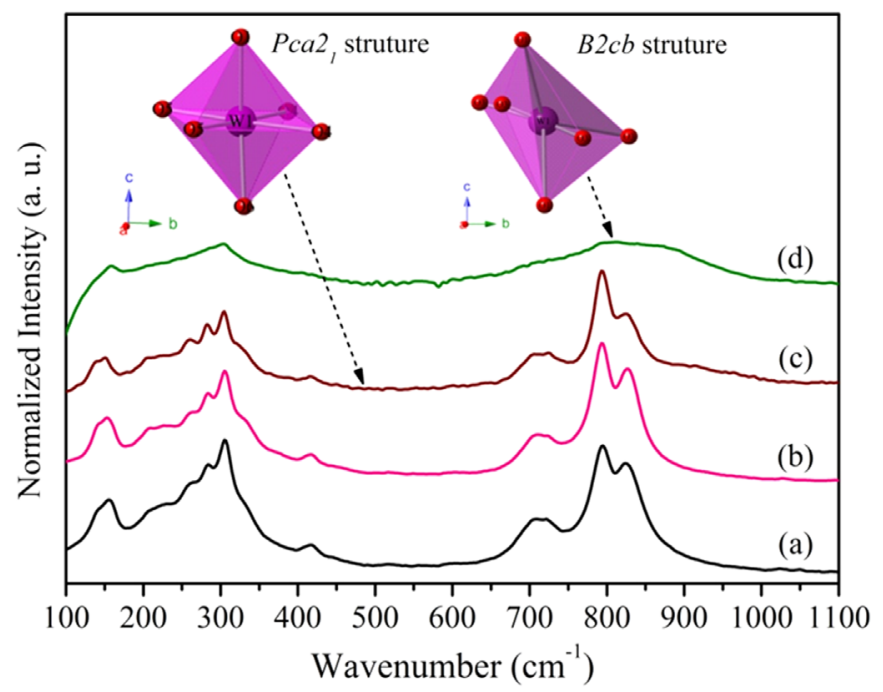

Fig. 2. Raman spectra of the as-prepared $\mathrm{Bi}_{2} \mathrm{WO}_{6}$ samples. (a) $\mathrm{S} 1$ (no surfactant), (b) S2 (SDS), (c) S4 (S3 after annealing) and (d) S3 (PVP).

\section{$\mathrm{P} 2{ }_{1}$ ab Structure}

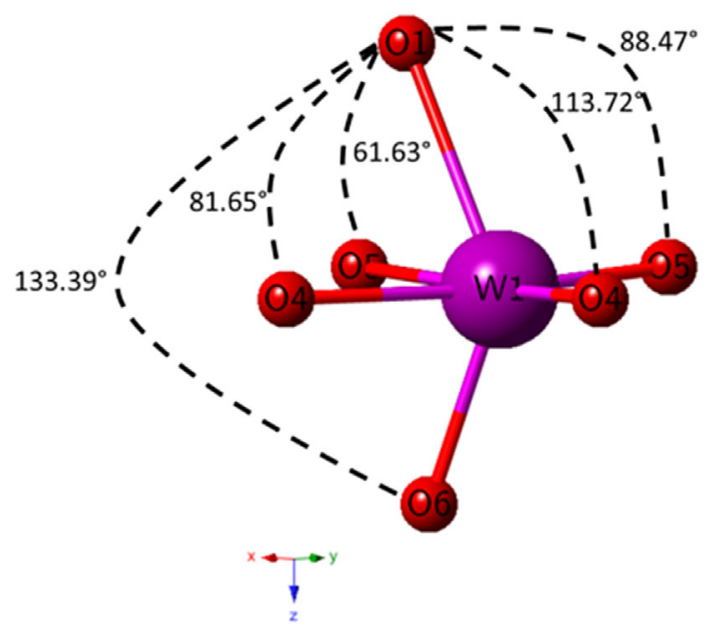

B2cb Structure

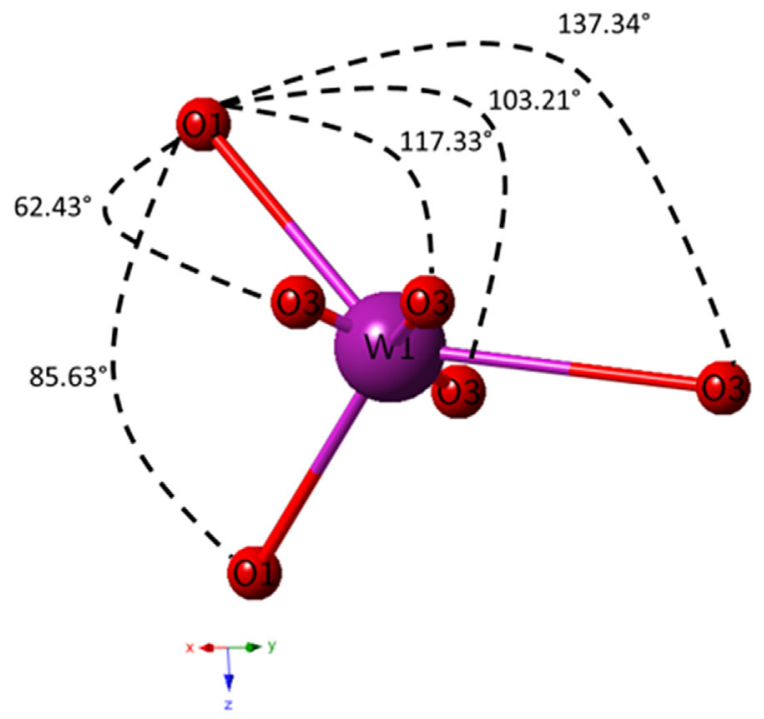

Fig. 3. Selected octahedral angles of $P 2{ }_{1} a b$ and $B 2 c b$ Structures. 


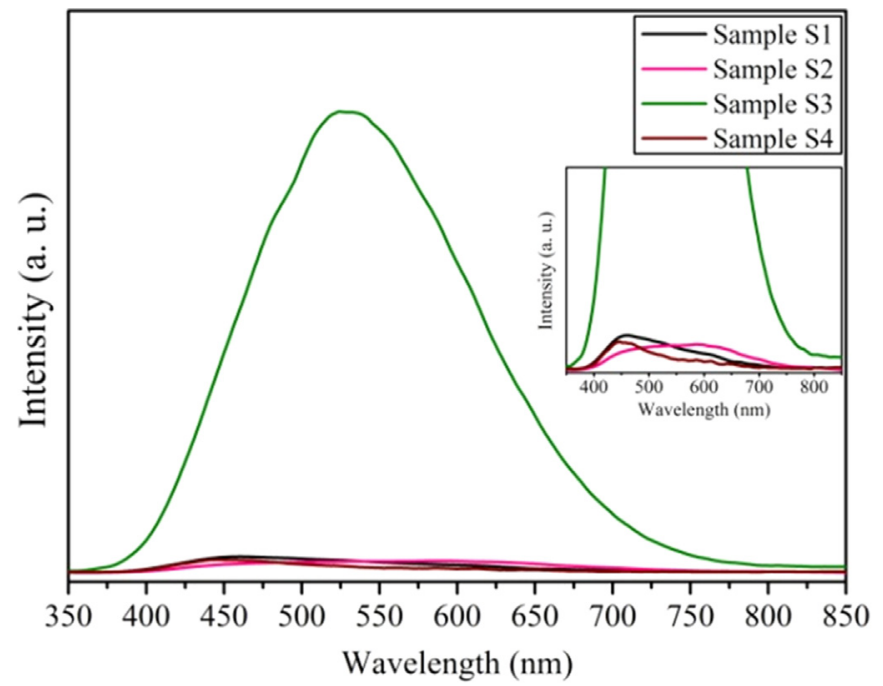

Fig. 4. Photoluminescence emission spectra of as-prepared sample of BWO excitation wavelength of $355 \mathrm{~nm}$.

distortions in the complex cluster, favor the breakdown of selection rules, having a consequence on the change in optical properties [20]. All these factors are responsible for defect creation, and can change the optical properties of metal tungstates (Fig. 3).

A peak at $430-530 \mathrm{~nm}$ is observed in the PL emission spectra of the BWO samples obtained in this work, as illustrated in Fig. 4. Sample S3 exhibits the highest PL intensity of all the BWO samples. This can be attributed to the change in the symmetry of the $\mathrm{Bi}_{2} \mathrm{WO}_{6}$ microcrystals $\left(P 2_{1} a b \rightarrow B 2 b c\right)$. In the metastable structure, the $\mathrm{WO}_{6}$ octahedra have a degree of distortion different to the usual $P 2_{1} a b$ structure (see in Fig. 3). This effect is better illustrated in refinement data found in the Supplementary material in Table SI 1 and SI 2. The higher PL intensity of the B2bc structure was confirmed by the PL spectrum of sample S4. A reduction in PL intensity was observed for sample $\mathrm{S4}$, which achieved similar intensities obtained with samples S1 and S2.

Therefore, it was confirmed that structural changes caused by templates in the reaction medium were responsible for structural modification, inducing the formation of the B2cb structure, which has a more intense photoluminescence than the $P 2{ }_{1} a b$ structure. This observation has not previously been described in the literature [21-23].

\section{Conclusion}

In summary, it has been successfully shown that sample S3, obtained by PVP surfactant-assisted hydrothermal synthesis exhibits a large enhancement in PL intensity, greater than that for BWO formed without surfactant and with SDS. The increased PL intensity is ascribed to a change of the crystal structure, induced by the surfactant template.

\section{Acknowledgment}

We acknowledge financial support from the FAPEMA (UNIVERSAL-00563/15, UNIVERSAL-00656/15, INFRA-03965/15), FUNCAP, CAPES and UFMA.

\section{Appendix A. Supporting information}

Supplementary data associated with this article can be found in the online version at http://dx.doi.org/10.1016/j.matlet.2016.08. 041 .

\section{References}

[1] N. Zhang, R. Ciriminna, M. Pagliaro, Y.J. Xu, Chem. Soc. Rev. 43 (15) (2014) 5276-5287.

[2] J. Zhang, Y. Wang, S. Li, X. Wang, F. Huang, A. Xie, Y. Shen, vol. 13(19), 2011, pp 5744-5750.

[3] H. Djani, P. Hermet, P. Ghosez, J. Phys. Chem. C 118 (25) (2014) 13514-13524.

[4] T. Zeng, X.T. Yu, S.P. Hui, Z.Y. Zhou, X.L. Dong, Mater. Res. Bull. 68 (2015) $271-275$.

[5] X. Ding, K. Zhao, L.Z. Zhang, Environ. Sci. Technol. 48 (10) (2014) 5823-5831.

[6] R.M. Silva, D.A.B. Barbosa, Cd.J.S. Mendonça, J.Rd.O. Lima, F.C. Silva, E. Longo, A P. Maciel, C.Wd.A. Paschoal, M.A.P. Almeida, J. Phys. Chem. Solids 96-97 (2016) 83-91.

[7] B. Wang, H. Yang, T. Xian, L.J. Di, R.S. Li, X.X. Wang, J. Nanomater. (2015) 146327.

[8] Z. Liu, X.Z. Liu, D.Z. Lu, P.F. Fang, S.J. Wang, Mater. Lett. 130 (2014) 143-145.

[9] W.W. Moses, Nucl. Instrum. Methods Phys. Res. Sect. A-Accel. Spectrom. Detect. Assoc. Equip. 487 (1-2) (2002) 123-128.

[10] Z.J. Zhang, W.Z. Wang, D. Jiang, J.Y. Xu, Catal. Commun. 55 (2014) 15-18.

[11] A. Singh, D.P. Dutta, M. Roy, A.K. Tyagi, M.H. Fulekar, J. Mater. Sci. 49 (5) (2014) 2085-2097.

[12] L. Zhang, C. Baumanis, L. Robben, T. Kandiel, D. Bahnemann, Small 7 (19) (2011) 2714-2720.

[13] S.O. Alfaro, A. Martinez-de la Cruz, Appl. Catal. A-Gen. 383 (1-2) (2010) $128-133$.

[14] M.A.P. Almeida, L.S. Cavalcante, C. Morilla-Santos, C.J. Dalmaschio, S. Rajagopal, M.S. Li, E. Longo, CrystEngComm 14 (2012) 7127-7132.

[15] Y.M. Cui, H.Q. Li, W.S. Hong, S.H. Fan, L.J. Zhu, Powder Technol. 247 (2013) 151-160.

[16] W. Wei, J.M. Xie, X.M. Lu, P.B. Osei, Z.X. Yan, S.C. Meng, H.L. Cui, Mon. Chem 145 (1) (2014) 47-59.

[17] Y. Liu, J. Goebl, Y. Yin, Chem. Soc. Rev. 42 (2013) 2610-2653.

[18] H.W. Huang, K. Liu, K. Chen, Y.L. Zhang, Y.H. Zhang, S.C. Wang, J. Phys. Chem. C 118 (26) (2014) 14379-14387.

[19] C.E. Housecroft, A.G. Sharpe, Inorganic Chemistry, in: P.E. Limited (Eds.), Inorg. Chem., England, 2008, p. 660.

[20] E. Longo, D.P. Volanti, V.M. Longo, L. Gracia, I.C. Nogueira, M.A.P. Almeida, A. N. Pinheiro, M.M. Ferrer, L.S. Cavalcante, J. Andres, J. Phys. Chem. C 118 (2) (2014) 1229-1239.

[21] C.M. Li, G. Chen, J.X. Sun, H.J. Dong, Y. Wang, C. Lv, Appl. Catal. B-Environ. 160 (2014) 383-389.

[22] N. Tian, Y.H. Zhang, H.W. Huang, Y. He, Y.X. Guo, J. Phys. Chem. C 118 (29) (2014) 15640-15648.

[23] Y. Yan, Y.F. Wu, Y.T. Yan, W.S. Guan, W.D. Shi, J. Phys. Chem. C 117 (39) (2013) 20017-20028. 